\title{
Winter Is Coming: How Humans Forage in a Temporally Structured Environment
}

\section{Citation}

Fougnie, Daryl, Sarah M Cormiea, Jinxia Zhang, George A Alvarez, and Jeremy M Wolfe. "Winter Is Coming: How Humans Forage in a Temporally Structured Environment." Journal of Vision 15, no. 11 (2015): 1-11.

\section{Permanent link}

http://nrs.harvard.edu/urn-3:HUL.InstRepos:41361467

\section{Terms of Use}

This article was downloaded from Harvard University's DASH repository, and is made available under the terms and conditions applicable to Other Posted Material, as set forth at http:// nrs.harvard.edu/urn-3:HUL.InstRepos:dash.current.terms-of-use\#LAA

\section{Share Your Story}

The Harvard community has made this article openly available.

Please share how this access benefits you. Submit a story.

Accessibility 


\title{
Winter is coming: How humans forage in a temporally structured environment
}

\author{
Daryl Fougnie
}

\author{
Sarah M. Cormiea
}

Science Division, New York University Abu Dhabi, Abu Dhabi, UAE Department of Psychology, Harvard University, Cambridge, MA, USA

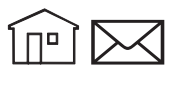

Department of Psychology, Harvard University, Cambridge, MA, USA

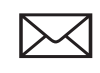

Visual Attention Lab, Brigham and Women's Hospital, Cambridge, MA, USA

School of Computer Science and Engineering, Nanjing University of Science and Technology, Nanjing, China

\author{
George A. Alvarez
}

\section{Jeremy M. Wolfe}

\author{
Department of Psychology, Harvard University, \\ Cambridge, MA, USA \\ Visual Attention Lab, Brigham and Women's Hospital, \\ Cambridge, MA, USA
Cisual Attention Lab, Brigham and Women's Hospital, \\ Harvard Medical School, Boston, MA, USA

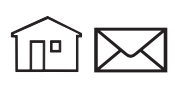

Much is known about visual search for single targets, but relatively little about how participants "forage" for multiple targets. One important question is how long participants will search before moving to a new display. Evidence suggests that participants should leave when intake drops below the average rate ("optimal foraging," Charnov, 1976). However, the real world has temporal structure (e.g., seasons) that could influence behavior. Does it matter if winter is coming and the next display will be worse than the last? We gave participants a series of search displays and asked them to collect targets as fast as possible. Target density was structured-rising and falling systematically across trials. We measured the duration for which participants foraged in each display (trials were terminated by participants). Foraging behavior was affected by temporal structure-counter to a simple optimal foraging account, observers searched displays longer when quality was falling compared to rising (Experiments 1 and 2). Additionally, we found that temporal structure altered explicit predictions about display quality (Experiment 2). These results demonstrate that foraging theories need to consider richer models of observers' representations of the world.

\section{Introduction}

In our daily lives, we are constantly faced with the problem of spotting items of interest in a complex visual environment. Over the last fifty years, a considerable amount of research has explored how we find such target items in visual displays containing distractor items (e.g., Chelazzi, Miller, Duncan, \& Desimone, 1993; Duncan \& Humphreys, 1989; Eckstein, 1998; Eriksen \& Schultz, 1979; Klein, 1988; Neisser, 1964; Palmer, 1990; Treisman \& Gelade, 1980; Wolfe, 1994, 1998, 2003; Wolfe, Cave \& Franzel, 1989; Woodman \& Luck, 1999). We now understand a great deal about this type of visual search. But searching for a particular target item (e.g., where did I leave my keys?) is only one example of the set of visual search problems that we face on a daily basis. Often we are not trying to find a single item, but are searching for a class of items of unknown quantity in our environment (e.g., picking ripe apples from an orchard or finding items of interest at a market). These types of search raise questions that have been rarely studied in classic visual

Citation: Fougnie, D., Cormiea, S. M., Zhang, J., Alvarez, G. A., \& Wolfe, J. M. (2015). Winter is coming: How humans forage in a temporally structured environment. Journal of Vision, 15(11):1, 1-11, doi:10.1167/15.11.1. 
search paradigms. For example, if I am picking apples from a particular tree in an orchard, at what point do I quit searching the current tree and move onto the next? Such questions are important, not only because they address an understudied area of visual search, but also because they explore the interactions between decisionmaking and visual cognition.

Decision-making in visual search tasks has been explored by studying the foraging behavior of animals (e.g., Charnov, 1976; Hayden, Pearson, \& Platt, 2011; McNamara, 1982; Mellgren, 1982; Stephens \& Krebs, 1986; Wajnberg, Fauvergue, \& Pons, 2000; Ydenberg, 1984) and by studying human behavior in laboratory foraging tasks (e.g., Cain, Vul, Clark, \& Mitroff, 2012; Wolfe, 2013; Hutchinson, Wilke, \& Todd, 2008; Pirolli, 2007). One major class of theories, termed Optimal Foraging Theory, provides an overarching theoretical framework for deciding when to quit searching a display (Charnov, 1976; Stephens \& Krebs, 1986). One influential idea in optimal foraging theory, Marginal Value Theorem, suggests that we measure the rate of return (e.g., how many apples I am collecting per unit of time) and that we quit searching the current display when the rate of return falls below the average rate of return (for all displays so far) (Charnov, 1976;

Mellgren, 1982; Stephens \& Krebs, 1986; Wajnberg et al., 2000; Ydenberg, 1984). Marginal Value Theorem correctly predicts that if we increase travel time (e.g., increasing the distance between trees in an orchard) that people will search longer before moving onto a new display. Targets cannot be acquired during travel, so increased travel decreases the average rate. Thus, people search longer because it takes longer for the current rate to fall to the average rate.

One of the appeals of Marginal Value Theorem is its simplicity - it requires an individual to keep track of only two pieces of information: the rate of target acquisition within the current patch and across all patches. However, the minimalistic nature is also a liability. The theory leaves no room for an understanding of the environment to influence behavior (such as its higher-order structure, Cain et al., 2012). Other approaches within Optimal Foraging Theory build on Marginal Value Theorem by proposing that individuals are building predictions of display/patch quality while foraging (Cain et al., 2012; McNamara, 1982; Olsson \& Brown, 2006). However, these accounts still propose that foraging behavior is driven by very limited information. In particular, the accounts ignore the knowledge and beliefs individuals have about objects or the environment.

Optimal foraging models have largely been tested in tasks where trials are drawn from a randomized design matrix. Critically, such designs minimize the contextual information provided by objects and events, and result in an artificial and unrealistic environment. The real world has structure - inferences about the nature of the world can come from a variety of sources (e.g., foraging in an apple orchard will be more successful in autumn than winter).

One important source of structure often ignored by optimal foraging models is temporal context. Many psychological processes have well-studied hysteresis effects (current performance depends on past input). For example, several paradigms reveal strong effects of a previous trial on performance (e.g., priming of popout: Maljkovic \& Nakayama, 1994, 1996, 2000; attentional capture: Lamy, Carmel, Egeth, \& Leber, 2006; Leber \& Egeth, 2006a, 2006b; Leonard \& Egeth, 2008; attentional blink: Potter, Chun, Banks, \& Muckenhoupt, 1998; working memory: Huang \& Sekuler, 2010; taskswitching: Schneider \& Logan, 2005). The perception of ambiguous or impoverished visual input reveals evidence that perception is an active and predictive process where recent experience influences current perceptual processing (Bar, 2007; Brascamp, et al., 2008; Corbett, Fischer, \& Whitney, 2011; Fischer \& Whitney, 2014; Liberman, Fischer, \& Whitney, 2014). Because the immediate past is a better predictor of the present than the more distant past, experiential effects fade over time (Corbett et al., 2011; Fischer \& Whitney, 2014).

In the present studies, we explored how foraging is influenced by temporal structure. We created historical dependencies between trials by adding "seasons" to foraging tasks. As participants advanced through trials, the displays would alternate between periods of plenty (many targets) and periods of scarcity (few targets), in a systematic fashion. Of interest is whether foraging behavior depends on whether participants are in rising (scarcity-to-plenty) or falling phases (plenty-to-scarcity).

To preview our results, we found that temporal history influenced foraging behavior in a foraging task for Ts among Ls (Experiment 1) - Participants foraged longer during falling phases. Note that these findings are inconsistent with classic optimal foraging theories. Quitting rules such as Marginal Value Theorem predict the opposite pattern; people should quit sooner in falling phases. The rate of target collection has been relatively high during the immediately preceding period of plenty. This raises the average rate of target collection. Thus, in the current patch with its lower quality, people should quit more quickly as the rate of return falls below the average.

Since we find that people search longer as patch quality falls, we will argue that participants are using temporal context to infer display quality. Searching for Ts among Ls is challenging, and participants will gain only limited information about display quality by scanning items. If a participant checks a handful of items and finds no targets, is this a bad display or bad 


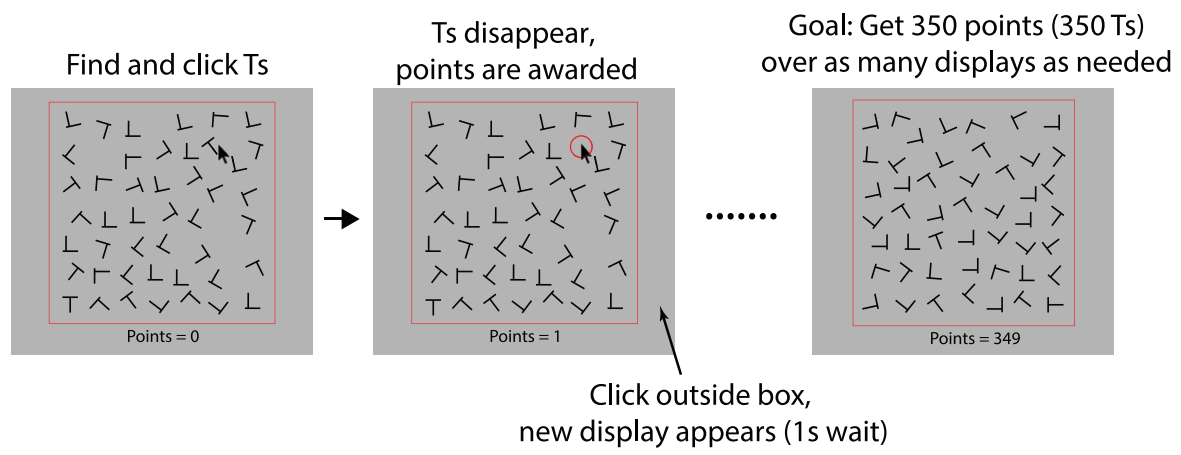

Figure 1. Methods for Experiment 1: Participants searched for Ts among Ls. Both letters were presented in a $7 \times 7$ display with jittered locations and randomly oriented letters. Displays contained 0-10 targets. Participants could quit foraging a trial by clicking outside the search frame. The red circle in the second panel is for illustration purposes to indicate the location of a clicked T. Participants did not see any red circles.

luck? The visual system uses recent experience to constrain uncertainty (Fischer \& Whitney, 2014). Therefore, temporal history may alter foraging behavior by altering participants' beliefs about display quality. People may forage for longer in falling phases than rising phases because the expected yield of search is influenced by the content of the previous displays (which is higher for falling phases), albeit not in the manner predicted by the Marginal Value Theorem.

Does temporal history alter explicit beliefs about display quality? In Experiment 2, on a subset of trials, we interrupted the foraging task and asked participants to directly rate display quality. We replicated the effect of temporal history on foraging behavior-people searched longer during falling than rising phases. Additionally, we found higher quality ratings for displays during falling phases, even though display quality was equivalent in falling and rising phases. Thus, temporal history alters both behavior and beliefs, consistent with an active inference (or Bayesian) account of foraging. Taken together, these results highlight the limitations of existing models and demonstrate that foraging theories need to consider richer models of observers' representations of the world.

\section{Experiment 1-Evidence for temporal context effects}

\section{Methods}

In this study, participants performed a simple foraging task, collecting Ts among Ls. Versions of this $\mathrm{T}$ among Ls task have been used extensively to study visual search (e.g., Wolfe, Palmer, \& Horowitz, 2010) but unlike most studies, our task involved searching for more than one target. As many as 10 targets were presented in a single display. Participants were not asked to find all targets in each display, but were instead asked to find as many targets as possible over many displays; thus, attempting to maximize their rate. Participants could quit a display at any time and wait $1 \mathrm{~s}$ for the next display.

\section{Stimuli}

Participants were asked to find a total of 350 Ts in displays comprised of Ts and Ls at random orientations (Figure 1). Between 0 and 10 targets were presented per display. Each display consisted of 49 items each subtending $0.9^{\circ} \times 0.9^{\circ}$ visual angle and presented at jittered locations within an invisible $7 \times 7$ grid $\left(15.7^{\circ} \times 15.7^{\circ}\right)$ presented at the center of the screen. The minimum distance between two stimuli was $0.75^{\circ}$. The current score was shown below the $\mathrm{T}$ and $\mathrm{L}$ display. To make the search process more difficult, the vertical line of the "L" stimuli was slightly to the right of a characteristic L, increasing the similarity with the $\mathrm{T}$ stimuli (Figure 1).

\section{Temporal structure}

The measure of interest is whether the foraging duration differs between "rising" and "falling" phases in the series of displays. The number of targets varied in a systematic fashion, determined largely by display order. To create "seasons," the number of targets varied between one and nine targets over a period of 40 trials in a sinusoidal manner. The sinusoid started in the rising phase for each participant. Pilot studies found no difference in foraging patterns if the phase of the sinusoid was initially in a rising or falling phase (see also Zhang, Gong, Fougnie, \& Wolfe, 2015). In practice, this sine wave was a step function (you cannot have 4.2 targets; see solid line in Figure 2). Moreover, the actual number of target presented was jittered by 


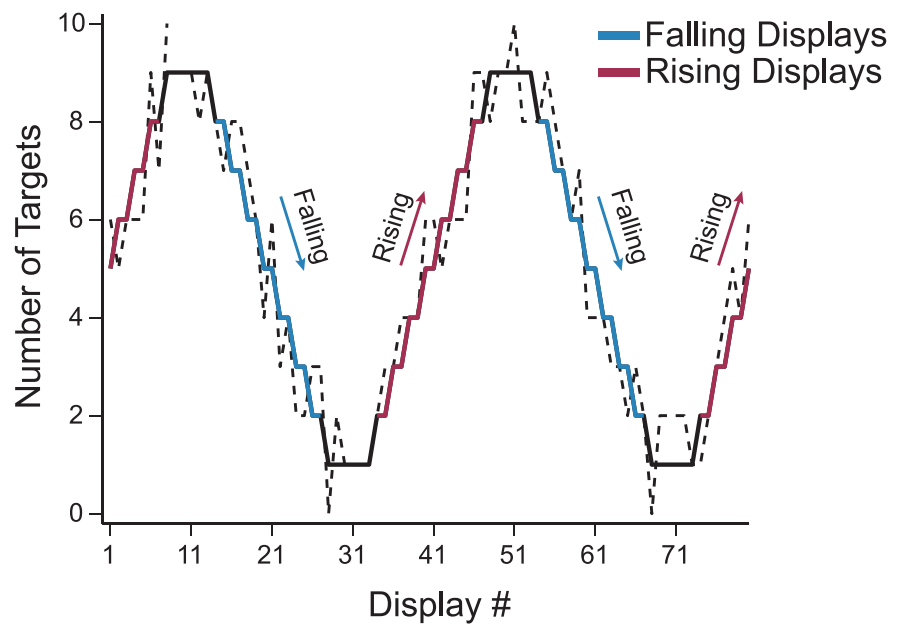

Figure 2. Target density varied systematically In Experiment 1 via a quantized sine wave pattern between one and nine targets over a period of 40 displays (Solid Line). Target density increased during rising phases (red) and decreased during falling phases (blue). For each participant, we included a oneitem jitter on target number. The dashed line is a representative example of what one participant might experience. The 40period temporal structure repeated until the participant completed the study, with independent jitter for each display.

one item. Thus, a display that would have held five targets could have four, five, or six targets). The dotted line in Figure 2 highlights an example of how target number could change over the first 80 trials of the study. Importantly, the total number of displays was not preset, but was determined by the number of selections it took a participant to reach 350 points.

\section{Scoring}

Participants collected Ts by moving the mouse to the location of a letter and clicking. If this item was a $\mathrm{T}$, one point would be awarded and that $\mathrm{T}$ would disappear. If an $\mathrm{L}$ was clicked in error, the point counter would decrease by 2 . Errors seldom occurred for any participants in any condition (less than $2 \%$ of responses). Participants could leave a display at any point by clicking outside a frame in which the items were presented. When a participant opted for a new display, the current search display would clear. After a "travel time" of $1 \mathrm{~s}$, a new display of 49 items would appear. Participants were explicitly told that it was ok to leave targets behind, and that their only goal was to collect as many Ts as possible per unit time. We used a self-terminating experiment (the experiment would end after 350 points were collected) to strongly motivate participants to collect Ts as fast as possible. Participants were told that the experiment would end once a certain number of points were reached, but were not told the exact number. The experiment concluded at the

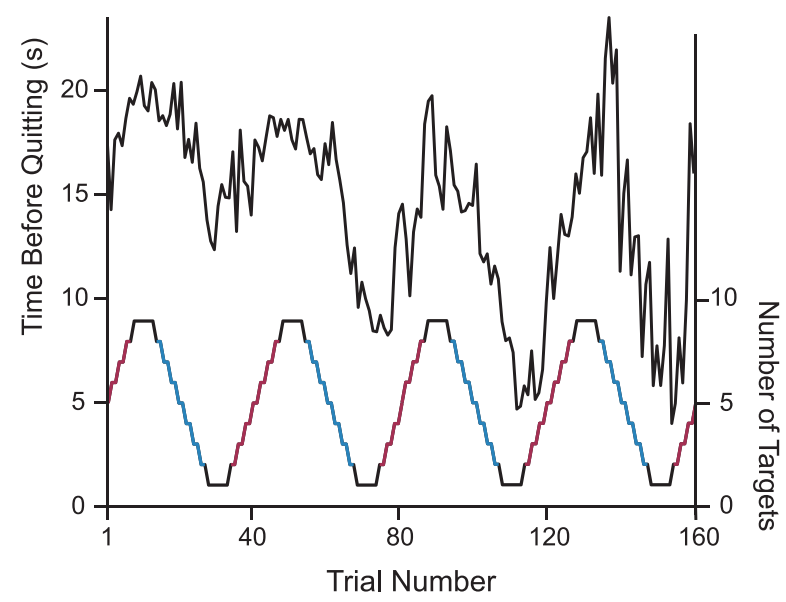

Figure 3. Time before quitting on each trial (black line; averaged across participants) for the first four periods of the temporal pattern of target density (red and blue step function). Participants foraged longer in displays with higher target density.

end of a display once at least 350 points were earned. Participants were given a chance to take a break every 15 displays.

\section{Participants}

Twelve participants were tested. They were between the ages of 18 and 28 years, had normal or correctedto-normal vision and received either $\$ 10$ per hour or course credit. The studies were performed in accordance with the Declaration of Helsinki and with Harvard University regulations. Data from one participant was excluded because that participant did not view enough different displays (the cutoff was 80 , or two full cycles, and the participant only viewed 62 displays).

\section{Results and discussion}

We measured the time participants foraged in each display (the time between display onset and offset). Figure 3 shows average foraging times as a function of trial number. We tested for effects on the number of targets in the display by conducting an ANOVA on foraging duration with target number as a factor. Unsurprisingly, foraging duration was heavily determined by the number of targets in the display $F(10$, $100)=24.35, p<0.001$.

More importantly, we asked whether temporal context influenced foraging duration. We split displays into falling and rising phases according to whether they were on the decreasing or increasing portion of the sinusoid that determined target quantity (Figure 2). Displays where the target quantity step function was at 

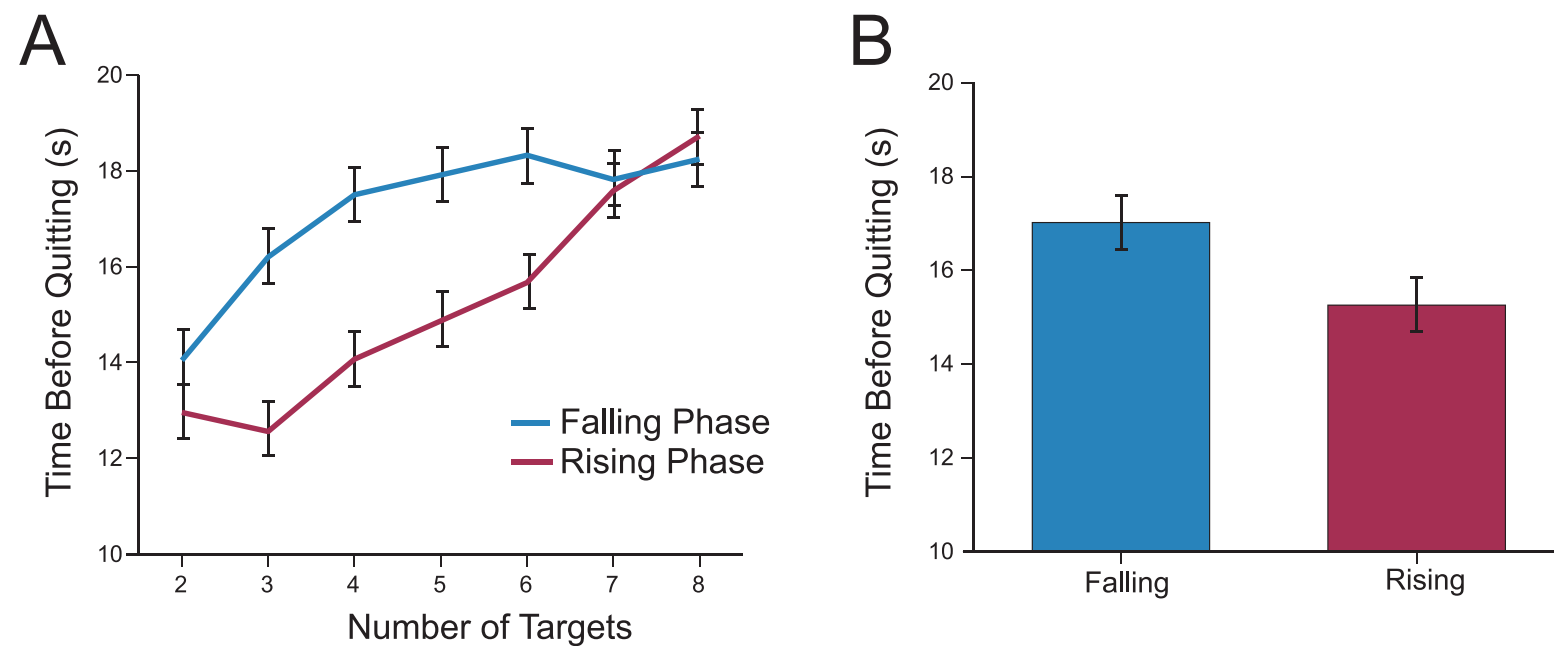

Figure 4. (A) Time before quitting in Experiment 1 for falling (blue) and rising (red) period as a function of the number of targets (before jitter). (B) The average foraging duration was longer for falling than rising phases. Error bars in panels A and B represent standard error of the difference in foraging duration between falling and rising displays.

ceiling or floor were not included because these trials are ambiguously associated with rising / falling phases. Rising and falling displays had equivalent numbers of targets, even accounting for target jitter $(p=0.88)$, and differed only in temporal context. Figure 4A plots the quitting time for rising and falling phases as a function of target quantity (before jitter). We found that participants foraged longer during falling than rising phases (Figure 4B), $t(10)=2.73, p=0.021$, Cohen's $d=$ 0.26 (paired $t$ test). ${ }^{1}$ A similar result was found when we compared the number of targets found per trial between falling (4.14) and rising (3.63) phases, $t(10)=$ $5.54, p<0.01$, Cohen's $d=0.73$. This effect was not significantly different from the foraging duration effect $(p=0.15)$. In addition, equivalent qualitative results were found for all subsequent comparisons of rising versus falling phases suggesting that the two measures are tapping the same construct. For brevity, we report foraging duration measures for subsequent analyses.

This study features a reliable temporal structure in the form of 'seasons' that created falling and rising phases. Is the large-scale temporal structure necessary or is it merely that performance on the current patch depends on the immediately preceding patch? To explore this we conducted a new study on 10 naïve participants using the same task as Experiment 1 but without temporal structure. Trials were categorized as "rising" or "falling" based on whether the $N$ th display had greater (rising phase) or fewer (falling phase) targets than the $N-1$ display. To address the problem of differences in target quantity between rising and falling conditions (trials where the $N$ th display has fewer targets than the $N-1$ display are more likely to be trials with a low target count), we computed separate averages based on target quantity (only trials with three to seven targets were included in the analysis) and averaged these conditions within participants. We found no difference between rising and falling phases, $t(9)=1.6, p=0.14$, using a paired $t$ test. This null finding does not preclude the possibility that the previous trial influences foraging behavior. Indeed, a recent study (Zhang et al., 2015) did observe small hysteresis effects in a foraging task with a randomized trial design. However, it does appear that the effects of temporal structure are cumulative over several patches.

We also explored whether participants explicitly noticed the temporal structure and whether this played a role in determining the temporal effects. During participant debriefing, participants were asked a series of three questions to determine whether they noticed a temporal pattern. We first asked participants if they noticed anything that they want to report. Second, we asked participants if they noticed any patterns. Finally, we asked participants if they noticed any temporal patterns. Only five participants noticed a pattern and those five could only report that target quality had streaks of scarcity and plenty. The differences in foraging duration for rising and falling phases were not reliably different for noticers and nonnoticers (the difference between average foraging durations for falling versus rising phases was 2.07 s for noticers $( \pm$ $1.26 \mathrm{~s}$ standard error) and $1.17 \mathrm{~s}( \pm 0.31 \mathrm{~s}$ standard error) for nonnoticers. An independent samples $t$ test found no difference between these numbers, $t(9)=0.76$, $p=0.47)$. With only five noticers and six nonnoticers and with substantial variability between participants, this study may lack the power to determine if awareness of the temporal pattern had an effect on performance. In any case, these results do suggest that being aware of the temporal pattern did not play a decisive role in driving the effect. 
Participants foraged longer during falling than during rising displays, even though the displays were equivalent in everything but temporal structure. Classic optimal foraging theories cannot explain such temporal effects. Why are participants inclined to forage longer during falling phases? Here we consider two broad possibilities. One possibility is that people's beliefs about the potential return of a display may be biased by the experience of the recent past. As participants forage they only gain partial information - there is a great deal of uncertainty about the likely number of targets in the display. When confronted with uncertainty, our visual system may use recent past experience to form predictions about the world (Bar, 2007; Fischer \& Whitney, 2014). On this account, if recent displays had few Ts, then the current display probably contains only a few Ts. If the prior displays were rich, it would be worth staying a little longer in the current display to look for the Ts that ought to be present there.

Another possibility is that temporal structure is not altering people's beliefs about the potential of a display, but that participants are making strategic decisions to maximize returns over the short-term. This account assumes that participants notice (either implicitly or explicitly) that the quality of displays is rising or falling. If participants are maximizing rewards for the immediate future, the potential reward of future displays could influence foraging time of the current display. For example, if a participant senses that subsequent displays will be worse than the current display, it would be reasonable to spend a bit more time extracting the relative riches from the current display.

A key distinction between these accounts is whether temporal history is altering participants' beliefs about displays. We tested this in Experiment 2 by interrupting the foraging task on a subset of trials and asking participants to make judgments about the target density of the current display. Moreover, we used quite a different foraging task in Experiment 2, allowing us to determine if the results of Experiment 1 generalize. To preview our results, we replicated the basic findings of Experiment 1 and established that temporal context alters both foraging behavior and beliefs about the world.

\section{Experiment 2-Temporal context alters beliefs about display quality}

The goal of Experiment 2 was to determine whether temporal context alters participants' beliefs about the number of targets in a display. To achieve this aim, this experiment differed from Experiment 1 in a number of respects. The principle difference was that, on a subset of trials, the foraging display was replaced by a screen asking participants to estimate the number of targets in the current display (including those targets as yet unseen and unclicked). In addition, we altered the foraging task to give us control over what items a participant saw in the displays. Instead of searching for targets in a crowded visual display, participants were shown items one at a time. Finally, the sinusoid (with a period of 40) that determined target number in Experiment 1 was replaced with a triangle wave (with a period of 12). This change was made because a sinusoid results in many displays that are near ceiling or floor, limiting the number of useful displays.

The goal of these changes to the task was to focus on the decisional aspects of foraging tasks. Variation in search processes presumably accounts for a large portion of variability in the efficiency with which participants found targets in Experiment 1. Deemphasizing search difficulty should therefore increase our ability to accurately measure decision processes. In addition, the changes in methodology allow us to test the generalizability of our effects by exploring whether they can be observed in an altered task. If a comparable difference between rising and falling displays were observed, this would suggest that the methodological changes did not disrupt the temporal effects observed in the first study.

\section{Methods}

We presumed that our measures would be noisier in this study due to the infrequency of target estimation trials and the reduction in the numbers of rising and falling phase displays. Consequently, we increased our participant sample size. The twenty participants were between the ages of 18 and 28 years, had normal or corrected-to-normal vision and received either $\$ 10$ per hour or course credit. In addition, participants were told that they could earn up to $\$ 10$ in bonus pay based on accuracy during the target estimation task. All participants were given the full $\$ 10$ bonus. The studies were performed in accordance with Harvard University regulations and approved by the Committee on the Use of Human Subjects in Research under the Institutional Review Board for the Faculty of Arts and Sciences. The data from all participants was included in the analyses because all participants completed at least two full cycles of temporal order.

The visual search component of the task was simplified (Figure 5). The display consisted of an $8 \times 8$ grid of locations ( $14^{\circ}$ visual angle). A subset (0-24) was assigned as target locations; the rest were distractor locations. Participants were not able to see the content of each location because each location was filled with a small black circle $\left(0.16^{\circ}\right)$. However, the content of a random location was revealed every $500 \mathrm{~ms}$. If the 


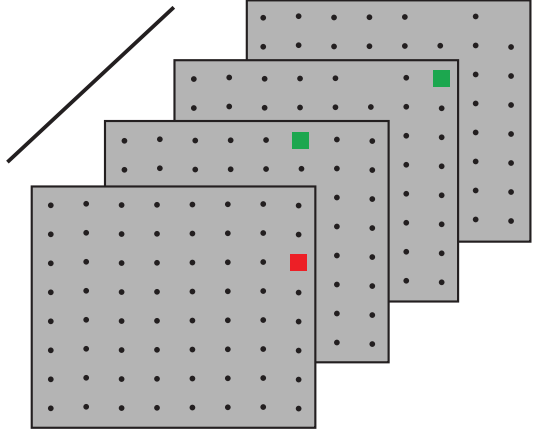

64 locations; $0-24$ green items Every $500 \mathrm{~ms}$ a location is revealed

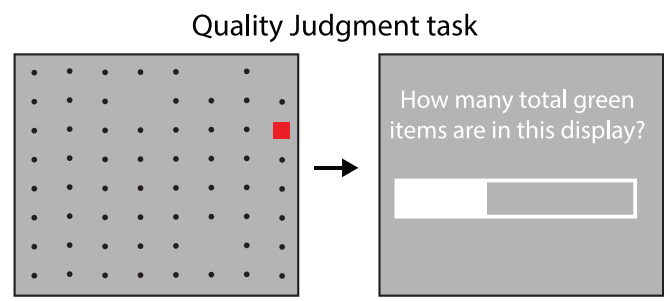

Trial was stopped after 0 or 16 reveals and replace by target estimation task

Figure 5. Methods for Experiment 2. Left: Every $500 \mathrm{~ms}$ a square was revealed from a random location with a location marker. If the location contained a target, the square was green (otherwise it was red). Participants were instructed to left-click the mouse to collect targets. Location markers with clicked targets were removed. Participants could terminate search at any point by right-clicking the mouse. Right: On a subset of trials, the foraging task was interrupted by a target estimation task. Participants were instructed to move a slider to the left or right to indicate the estimated proportion of targets in the 64-item display (including seen and unseen items). Participants were given practice with the slider task to ensure the onscreen instructions were understood.

revealed location was a target location, a green square appeared at the location $\left(0.47^{\circ}\right)$. If the location did not have a target, a red square appeared instead. Participants were instructed to click the left mouse button whenever a green square appeared. Participants did not have to click on the square: A click anywhere within the display would count. In addition, clicks counted if they were within 1s of the onset of the target, giving participants sufficient time for responses. The task required constant attention, but was not too demanding (less than $1 \%$ of targets were missed). A clicked square disappeared, along with its location marker. New locations were sampled randomly, with replacement, from all locations with a marker. Thus, as in visual search, there were diminishing returns for remaining in a display for long periods.

Participants could leave a patch any time by clicking the right mouse button. As in Experiment 1, participants were instructed to collect targets as fast as possible and that they were not required to find every target in a display. The experiment terminated when participants collected 350 points. Correct targets added 1 point. False alarms subtracted 2 points (false alarms were rare, $1.4 \%$ of responses).

At specific points in the temporal structure, the foraging task was interrupted, and replaced by a quality judgment screen. These interruptions occurred at trials with eight and 16 targets in rising and falling phases (Figure 6). The task was interrupted either at the start of a display, after onset of the $8 \times 8$ display of dots, or during foraging, after 16 items were shown. In the 16 -item reveal condition, the 16 chosen items were constrained to show two or four targets (equally often for both the eight and 16 target conditions). If participants quit foraging the display before the $16^{\text {th }}$ item was revealed, the quality judgment task was skipped (this happened infrequently, less than $3 \%$ of target estimation trials (2.8\% of falling phase trials; $2.4 \%$ of rising phase trials).

The quality judgment screen contained a white slider that filled from left to right. Participants were instructed that the proportion of the slider that was filled represented the estimated proportion of the display that functioned as targets (including seen and unseen items). Participants adjusted the slider by moving the mouse cursor left or right, and clicked the

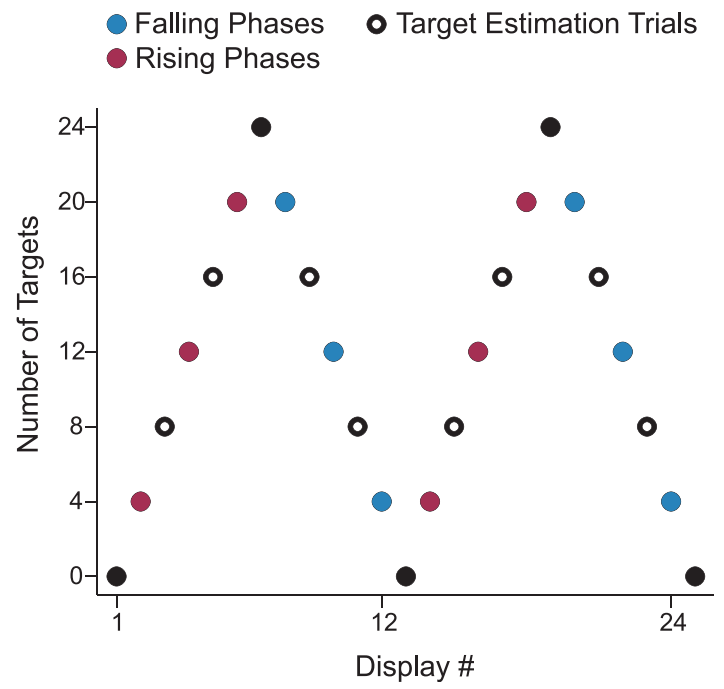

Figure 6. Target density varied systematically in Experiment 2 via a triangle wave pattern between 0 and 24 targets with a period of 12 displays. Target density increased during rising phases (red) and decreased during falling phases (blue). Hollow circles indicate trials that were interrupted by a target estimation judgment. 

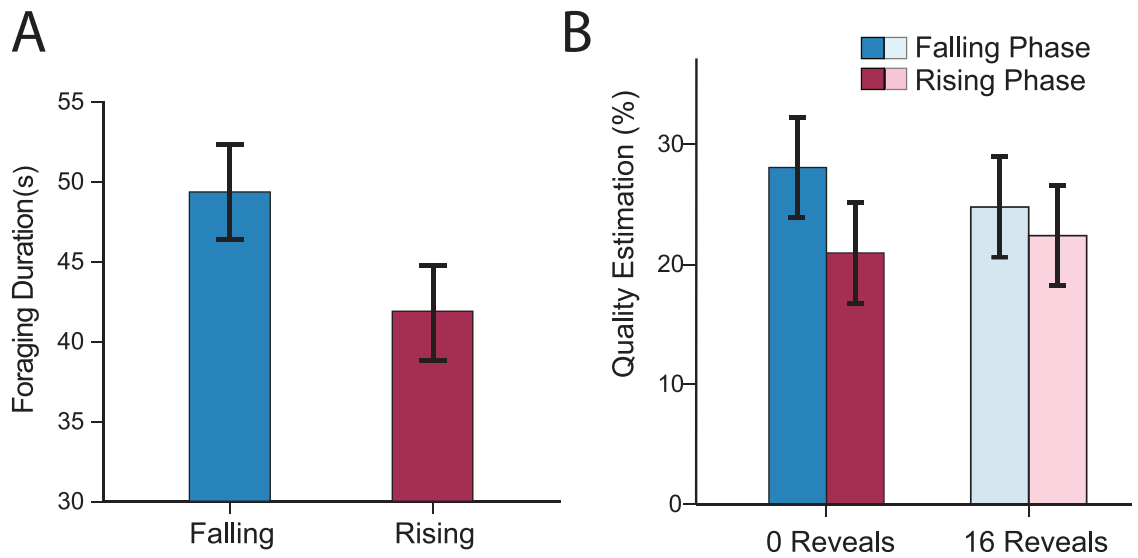

Figure 7. (A) Foraging duration for rising (red) and falling (blue) phases in Experiment 2. (B) Quality estimate percentages for the 0reveal (darker colors) and 16-reveal conditions (lighter colors) plotted for rising and falling phases. Error bars in panels $A$ and $B$ represent standard error of the difference in foraging duration or quality estimation between falling and rising displays.

mouse to submit a response. No feedback was provided after the response. Participants were instructed that accuracy in this task would be used to determine bonus monetary compensation, but were not provided any details on how this reward was calculated. In practice, all participants were awarded the $\$ 10$ bonus.

As in Experiment 1, temporal order varied in a systematic fashion. Here we used a triangle wave that varied between 0 and 24 targets with a period of 12 displays (each step changed target quantity by 4 items) (Figure 6). We no longer added jitter to target number - target density was completely determined by trial order. Jitter was removed because participants only saw a portion of any display, rendering the value of jitter minimal. Importantly, falling and rising phases were matched in target number.

\section{Results and discussion}

We tested for effects on the number of targets in the display by conducting an ANOVA on foraging duration with target number as a factor. Foraging duration was heavily determined by the number of targets in the display, $F(6,114)=28.86, p<0.001$. More importantly, we replicated the effect of temporal order: Participants searched longer during falling than rising periods, $t(19)=2.09, p=0.05$, Cohen's $d=0.32$ (Figure 7A). Thus, the effect of temporal structure appears to generalize across methodological changes such as whether the task requires guided search or whether items are revealed to participants. Additionally, we note that similar effects of temporal structure were also seen in an easier, nonguided search task (Zhang et al., 2015) further demonstrating that this effect is resilient across differences in methodology.

Do temporal structure effects at least partially reflect changes in participants' beliefs about display quality?
Figure 7B plots the average quality judgments for rising and falling phases in the 0 -item revealed and 16-item revealed conditions. Averaged across all conditions, participants estimated that $24.2 \%$ of items were targets (the equivalent of estimating 15.5 targets), which was close to the actual average of $18.8 \%$ (or 12 targets). This indicates that participants understood the task and were motivated to respond accurately. Quality estimates were entered into a $2 \times 2$ ANOVA with conditions of Items Revealed ( 0 or 16) and Temporal Period (Falling or Rising). We found a main effect of Temporal Period-participants judged displays as containing more targets during falling phases even though the target quality was equivalent for falling and rising phases, $F(1,19)=12.05, p<0.005$. We also examined whether revealing information about display quality influenced the strength of the temporal structure effect. If participants are using the past to constrain uncertainty, then temporal effects should be largest when participants have the most uncertainty about display quality. Consistent with this prediction, while we found no main effect of number of Reveals, $F(1,19)=0.15, p=0.7$, we did see an interaction between number of Reveals and Temporal Period, $F(1,19)=8.73, p<0.01$. This interaction likely indicates that participants were less influenced by temporal order when more information about the current display could guide their judgments.

Taken together, the results suggest that the temporal order effects seen here (as well as those seen by Zhang et al., 2015) are not driven just by expectations about an upcoming winter or spring, but at least in part by the past influencing participants' beliefs about the present. Note that this result does not rule out other potential influences of temporal order on foraging behavior, as there may be multiple contributions to this effect. 


\section{General discussion}

Many tasks that we need to perform on a daily basis require surveying an environment for items of interest and making decisions about how to maximize the number or quality of items that are obtained. The current work explores how decisions are made in visual cognition tasks using foraging as a case study. Which factors influence when you quit searching one display and move on to the next?

This question has been addressed in classic optimal foraging models by proposing that participants optimize input by leaving whenever the current intake drops below the average rate (Marginal Value Theorem). This simple rule has been highly influential because it provides an overarching framework that can be used to study everything from parasitic insects (Wajnberg et al., 2000), to birds (Ydenberg, 1984), to humans (Cain, et al., 2012; Wolfe, 2013). Further, this theory can explain behavior while proposing minimal cognitive machinery - the rule relies on basic arithmetic and forgoes the need for internal representations or theories about the world. But one important limitation in this approach is the assumption that foraging behavior can be explained by ignoring cognitive states. We are not passive observers to the world-perception is inferential (Brady \& Chun, 2007; Brady \& Tenenbaum, 2013; Chun \& Jiang, 1998; Feldman, Griffiths, \& Morgan, 2009; Fischer \& Whitney, 2014).

One reason for the success of classic optimal foraging theories is that they have been tested in tasks in which experimenter control and randomization strip structure and context from the world. In such cases, the limitations of this approach may not be apparent. Here we introduced temporal structure to the world by adding seasons. As participants advanced through the study, they experienced transitions from plenty-to-scarcity (falling) and scarcity-to-plenty (rising). In contrast to existing accounts, we found that participants would forage longer in falling than rising phases (Experiments 1 and 2). Furthermore, this temporal context effect not only altered foraging behavior, but was found to influence explicit judgments of target density (Experiment 2). Taken together, these findings argue that foraging behavior is driven not only by estimates of current and average intake, but by inferences about the current state of the world shaped by previous experience.

Our findings are consistent with other literature demonstrating how past experience can influence current perception, recognition, or memory through Bayesian inference (Crawford, Huttenlocher, \& Engebretson, 2000; Feldman et al., 2009; Fischer \& Whitney, 2014). Bayesian inference provides a natural account for how previous experience can influence foraging behavior. We come into a display with prior beliefs about the world. These prior beliefs are informed by experience. Consistent with this, the prior experience effect on estimates of target density (Experiment 2) became weaker when participants were given some experience of the current display. The mechanism by which prior beliefs combine with experience need not be explicitly Bayesian (Sailor \& Antoine, 2005; Crawford, Huttenlocher, \& Hedges, 2006), but Bayesian inference provides a compelling account for how this could be achieved.

There is a considerable amount of research on animal foraging behavior, but our research highlights an area that is not well understood. Studies show seasonal variation in foraging patterns (e.g., Conner, 1981; Owen-Smith, 1994; van Marken Lichtenbelt, 1993), but do nonhuman animals use recent experience to guide foraging behavior? Studies have found that poor foraging experiences (e.g., low target quantity at a particular location) can lead to longer search durations in the subsequent foraging episode (Tentelier, Desouhant, \& Fauvergue, 2006; Visser, van Alphen, \& Nell, 1992), consistent with the predictions of a moment-tomoment optimal foraging model. But what would happen if predictable, short-term temporal signals were given to nonhuman animals? To our knowledge, no existing study directly addresses this question.

Our findings highlight the limitations of existing models. Classic optimal foraging models often give observers considerable information (such as the distribution of possible states of the world) but ignore how observers acquire this information (Charnov, 1976; Hutchinson, Wilke, \& Todd, 2008). Optimal foraging models may make good rules of thumb for approximating behavior, but they are only a starting point. It may be tempting to ignore context effects as nuances on theory. But context effects reveal the aspects of the environment that an observer uses to shape beliefs about the world. Foraging theories will need to account for these context effects. Here we show that foraging is altered by temporal context. Future work will no doubt reveal a rich tapestry of context effects. Understanding these context effects will give valuable insights on the interaction between vision and decision-making.

Keywords: visual search, foraging, temporal structure, hysteresis

\section{Acknowledgments}

We thank Krista Ehinger, Xue Gong, and Mathew Cain for helpful discussions. This work was supported by the National Science Foundation (CELEST NSF SBE-0354378) and the National Natural Science Fund of China (61233011).

Commercial relationships: none. 
Corresponding author: Daryl Fougnie.

Email: darylfougnie@gmail.com.

Address: Science Division, Psychology, New York University Abu Dhabi, Abu Dhabi, United Arab Emirates.

\section{Footnote}

${ }^{1}$ The experiment was self-terminating and therefore participants saw different numbers of displays. Could this explain the results? To test this, we ran a second analysis with only the first two cycles of the target quantity stepfunction, which all participants completed. We still found a difference between rising and falling phases, $t(10)=$ $2.59, p=0.027$, inconsistent with this possibility.

\section{References}

Bar, M. (2007). The proacti ve brain: Using analogies and associations to generate predictions. Trends in Cognitive Sciences, 11(7), 280-289.

Brady, T. F., \& Chun, M. M. (2007). Spatial constraints on learning in visual search: Modeling contextual cuing. Journal of Experimental Psychology: Human Perception and Performance, 33(4), 798.

Brady, T. F., \& Tenenbaum, J. B. (2013). A probabilistic model of visual working memory: Incorporating higher order regularities into working memory capacity estimates. Psychological Review, 120(1), 85.

Brascamp, J. W., Knapen, T. H., Kanai, R., Noest, A. J., Van Ee, R., \& Van Den Berg, A. V. (2008). Multi-timescale perceptual history resolves visual ambiguity. PloS One, 3(1), e1497.

Cain, M. S., Vul, E., Clark, K., \& Mitroff, S. R. (2012). A Bayesian optimal foraging model of human visual search. Psychological Science, 23(9), 10471054.

Charnov, E. L. (1976). Optimal foraging, the marginal value theorem. Theoretical Population Biology, 9(2), 129-136.

Chelazzi, L., Miller, E. K., Duncan, J., \& Desimone, R. (1993). A neural basis for visual search in inferior temporal cortex. Nature, (363), 345-347.

Chun, M. M., \& Jiang, Y. (1998). Contextual cueing: Implicit learning and memory of visual context guides spatial attention. Cognitive Psychology, 36(1), 28-71.

Conner, R. N. (1981). Seasonal changes in woodpecker foraging patterns. The Auk, 98(3), 562-570.

Corbett, J. E., Fischer, J., \& Whitney, D. (2011).
Facilitating stable representations: Serial dependence in vision. PloS One, 6(1), e16701.

Crawford, L. E., Huttenlocher, J., \& Engebretson, P. H. (2000). Category effects on estimates of stimuli: Perception or reconstruction? Psychological Science, 11(4), 280-284.

Crawford, L. E., Huttenlocher, J., \& Hedges, L. V. (2006). Within-category feature correlations and Bayesian adjustment strategies. Psychonomic Bulletin \& Review, 13(2), 245-250.

Duncan, J., \& Humphreys, G. W. (1989). Visual search and stimulus similarity. Psychological Review, 96(3), 433.

Eckstein, M. P. (1998). The lower visual search efficiency for conjunctions is due to noise and not serial attentional processing. Psychological Science, 9(2), 111-118.

Eriksen, C. W., \& Schultz, D. W. (1979). Information processing in visual search: A continuous flow conception and experimental results. Perception \& Psychophysics, 25(4), 249-263.

Feldman, N. H., Griffiths, T. L., \& Morgan, J. L. (2009). The influence of categories on perception: Explaining the perceptual magnet effect as optimal statistical inference. Psychological Review, 116(4), 752.

Fischer, J., \& Whitney, D. (2014). Serial dependence in visual perception. Nature Neuroscience, 17(5), 738 743.

Hayden, B. Y., Pearson, J. M., \& Platt, M. L. (2011). Neuronal basis of sequential foraging decisions in a patchy environment. Nature Neuroscience, 14(7), 933-939.

Huang, J., \& Sekuler, R. (2010). Distortions in recall from visual memory: Two classes of attractors at work. Journal of Vision, 10(2):24, 1-27, doi:10. 1167/10.2.24. [PubMed] [Article]

Hutchinson, J., Wilke, A., \& Todd, P. M. (2008). Patch leaving in humans: Can a generalist adapt its rules to dispersal of items across patches? Animal Behaviour, 75(4), 1331-1349.

Klein, R. (1988). Inhibitory tagging system facilitates visual search. Nature, 334(6181), 430-431.

Lamy, D., Carmel, T., Egeth, H. E., \& Leber, A. B. (2006). Effects of search mode and intertrial priming on singleton search. Perception \& Psychophysics, 68(6), 919-932.

Leber, A. B., \& Egeth, H. E. (2006a). It's under control: Top-down search strategies can override attentional capture. Psychonomic Bulletin \& Review, 13(1), 132-8.

Leber, A. B., \& Egeth, H. E. (2006b). Attention on autopilot: Past experience and attentional set. Visual Cognition, 14(4-8), 565-583. 
Leonard, C. J., \& Egeth, H. E. (2008). Attentional guidance in singleton search: An examination of top-down, bottom-up, and intertrial factors. Visual Cognition, 16(8), 1078-1091.

Liberman, A., Fischer, J., \& Whitney, D. (2014). Serial dependence in the perception of faces. Current Biology, 24(21), 2569-2574.

Maljkovic, V., \& Nakayama, K. (1994). Priming of pop-out: I. Role of features. Memory \& Cognition, 22(6), 657-672.

Maljkovic, V., \& Nakayama, K. (1996). Priming of pop-out: II. The role of position. Perception \& Psychophysics, 58(7), 977-991.

Maljkovic, V., \& Nakayama, K. (2000). Priming of popout: III. A short-term implicit memory system beneficial for rapid target selection. Visual Cognition, 7(5), 571-595.

McNamara, J. (1982). Optimal patch use in a stochastic environment. Theoretical Population Biology, 21(2), 269-288.

Mellgren, R. L. (1982). Foraging in a simulated natural environment: There's a rat loose in the lab. Journal of the Experimental Analysis of Behavior, 38(1), 93-100.

Neisser, U. (1964). Visual search. Scientific American, 210(6), 94-102.

Olsson, O., \& J. S Brown, (2006). The foraging benefits of information and the penalty of ignorance. Oikos, 112(2), 260-273.

Owen-Smith, N. (1994). Foraging responses of kudus to seasonal changes in food resources: Elasticity in constraints. Ecology, 75(4), 1050-1062.

Palmer, J. (1990). Attentional limits on the perception and memory of visual information. Journal of Experimental Psychology: Human Perception and Performance, 16(2), 332.

Pirolli, P. L. (2007). Information foraging theory: Adaptive interaction with information. Oxford, UK: Oxford University Press.

Potter, M. C., Chun, M. M., Banks, B. S., \& Muckenhoupt, M. (1998). Two attentional deficits in serial target search: The visual attentional blink and an amodal task-switch deficit. Journal of Experimental Psychology: Learning, Memory, and Cognition, 24(4), 979.

Sailor, K. M., \& Antoine, M. (2005). Is memory for stimulus magnitude Bayesian? Memory \& Cognition, 33(5), 840-851.

Schneider, D. W., \& Logan, G. D. (2005). Modeling task switching without switching tasks: A shortterm priming account of explicitly cued performance. Journal of Experimental Psychology: General, 134(3), 343.
Stephens, D. W., \& Krebs, J. R. (1986). Foraging theory. Princeton, NJ: Princeton University Press.

Tentelier, C., Desouhant, E., \& Fauvergue, X. (2006). Habitat assessment by parasitoids: Mechanisms for patch use behavior. Behavioral Ecology, 17(4), 515521.

Treisman, A. M., \& Gelade, G. (1980). A featureintegration theory of attention. Cognitive Psychology, 12(1), 97-136.

van Marken Lichtenbelt, W. D. (1993). Optimal foraging of a herbivorous lizard, the green iguana in a seasonal environment. Oecologia, 95(2), 246-256.

Visser, M. E., van Alphen, J. J., \& Nell, H. W. (1992). Adaptive superparasitism and patch time allocation in solitary parasitoids: The influence of pre-patch experience. Behavioral Ecology and Sociobiology, 31(3), 163-171.

Wajnberg, E., Fauvergue, X., \& Pons, O. (2000). Patch leaving decision rules and the Marginal Value Theorem: An experimental analysis and a simulation model. Behavioral Ecology, 11(6), 577-586.

Wolfe, J. M. (1994). Guided search 2.0 a revised model of visual search. Psychonomic Bulletin \& Review, 1(2), 202-238.

Wolfe, J. M. (1998). What can 1 million trials tell us about visual search? Psychological Science, 9(1), 33-39.

Wolfe, J. M. (2003). Moving towards solutions to some enduring controversies in visual search. Trends in Cognitive Sciences, 7(2), 70-76.

Wolfe, J. M. (2013). When is it time to move to the next raspberry bush? Foraging rules in human visual search. Journal of Vision, 13(3):10, 1-17, doi:10. 1167/13.3.10. [PubMed] [Article]

Wolfe, J. M., Cave, K. R., \& Franzel, S. L. (1989). Guided search: An alternative to the feature integration model for visual search. Journal of Experimental Psychology: Human Perception and Performance, 15(3), 419.

Wolfe, J. M., Palmer, E. M., \& Horowitz, T. S. (2010). Reaction time distributions constrain models of visual search. Vision Research, 50(14), 1304-1311.

Woodman, G. F., \& Luck, S. J. (1999). Electrophysiological measurement of rapid shifts of attention during visual search. Nature, 400(6747), 867-869.

Ydenberg, R. C. (1984). Great tits and giving-up times: Decision rules for leaving patches. Behaviour, 90(1/ 3), 1-24.

Zhang, J. Gong, X., Fougnie, D., \& Wolfe, J. M. (2015). Using the past to anticipate the future in human foraging behavior. Vision Research, 111(2015), 6674. 